Pacific Journal of Mathematics

TRIPLE SERIES EQUATIONS INVOLVING DAGUERRE 


\section{TRIPLE SERIES EQUATIONS INVOLVING LAGUERRE POLYNOMIALS}

\section{JOHN S. LOWNDES}

In this paper it is shown that the problem of solving the triple series equations of the first kind

$$
\begin{array}{ll}
\sum_{n=0}^{\infty} A_{n} \Gamma(\alpha+1+n) L_{n}(\alpha ; x)=0, & 0 \leqq x<a, \\
\sum_{n=0}^{\infty} A_{n} \Gamma(\alpha+\beta+n) L_{n}(\alpha ; x)=f(x), & a<x<b, \\
\sum_{n=0}^{\infty} A_{n} \Gamma(\alpha+1+n) L_{n}(\alpha ; x)=0, & b<x<\infty,
\end{array}
$$

and the triple series equations of the second kind

$$
\begin{array}{ll}
\sum_{n=0}^{\infty} B_{n} \Gamma(\alpha+\beta+n) L_{n}(\alpha ; x)=g(x), & 0 \leqq x<a, \\
\sum_{n=0}^{\infty} B_{n} \Gamma(\alpha+1+n) L_{n}(\alpha ; x)=0, & a<x<b, \\
\sum_{n=0}^{\infty} B_{n} \Gamma(\alpha+\beta+n) L_{n}(\alpha ; x)=h(x), & b<x<\infty,
\end{array}
$$

where $\alpha+\beta>0,0<\beta<1, L_{n}(\alpha ; x)=L_{n}^{\alpha}(x)$ is the Laguerre polynomial and $f(x), g(x)$ and $h(x)$ are known functions, can be reduced to that of solving a Fredholm integral equation of the second kind. The analysis is formal and no attempt is made to supply details of rigour.

In a recent paper [1] the present author has solved the dual series equations

$$
\begin{array}{ll}
\sum_{n=0}^{\infty} C_{n} \Gamma(\alpha+\beta+n) L_{n}(\alpha ; x)=f(x), & 0 \leqq x<d, \\
\sum_{n=0}^{\infty} C_{n} \Gamma(\alpha+1+n) L_{n}(\alpha ; x)=g(x), & d<x<\infty,
\end{array}
$$

where $\alpha+\beta>0,0<\beta<1$. The triple series equations (1) to (6) can be considered to be extensions of the equations (7) and (8). In other papers [2], [3], [5], and in a book by Sneddon [4], the solution of triple integral equations involving Bessel functions and triple series equations involving functions orthogonal on a finite interval have been considered and in every case the solution is expressed in terms of the solution of one or two Fredholm integral equations.

2. In the course of the analysis we shall use the following results. 
The orthogonality relation for Laguerre polynomials is

( 9 ) $\int_{0}^{\infty} x^{\alpha} e^{-x} L_{m}(\alpha ; x) L_{n}(\alpha ; x) d x=\frac{\Gamma(\alpha+1+n)}{\Gamma(n+1)} \delta_{m n}, \quad \alpha>-1$, where $\delta_{m n}$ is the Kronecker delta.

Using the results (5) and (20) given in [1] it is easily shown that

$$
\begin{aligned}
S(r, x) & =(r x)^{\alpha} \sum_{n=0}^{\infty} \frac{\Gamma(n+1) \Gamma(\alpha+\beta+n)}{\{\Gamma(\alpha+1+n)\}^{2}} L_{n}(\alpha ; x) L_{n}(\alpha ; r) \\
& =\frac{1}{\{\Gamma(1-\beta)\}^{2}} \int_{0}^{t} n(y)(r-y)^{-\beta}(x-y)^{-\beta} d y \\
& =\frac{1}{\{\Gamma(1-\beta)\}^{2}} S_{t}(r, x),
\end{aligned}
$$

where $\beta<1, \alpha+\beta>0, n(y)=e^{y} y^{\alpha+\beta-1}$ and $t=\min (r, x)$.

If $f(x)$ and $f^{\prime}(x)$ are continuous in $a \leqq x \leqq b$ and if $0<\sigma<1$, then the solutions of the Abel integral equations

$$
f(x)=\int_{a}^{x} \frac{F(y)}{(x-y)^{\sigma}} d y,
$$

and

$$
f(x)=\int_{x}^{b} \frac{F(y)}{(y-x)^{\sigma}} d y
$$

are given by

$$
F(y)=\frac{\sin (\sigma \pi)}{\pi} \frac{d}{d y} \int_{a}^{y} \frac{f(x)}{(y-x)^{1-\sigma}} d x,
$$

and

$$
F(y)=-\frac{\sin (\sigma \pi)}{\pi} \frac{d}{d y} \int_{y}^{b} \frac{f(x)}{(x-y)^{1-\sigma}} d x
$$

respectively.

3. Equations of the first kind. In order to solve the triple series equations of the first kind we set

$$
\sum_{n=0}^{\infty} A_{n} \Gamma(\alpha+1+n) L_{n}(\alpha ; x)=\phi(x), \quad a<x<b,
$$

and using the orthogonality relation (9) we find from equations (1), (16) and (3) 


$$
A_{n}=\frac{\Gamma(n+1)}{\{\Gamma(\alpha+1+n)\}^{2}} \int_{a}^{b} r^{\alpha} e^{-r} \phi(r) L_{n}(\alpha ; r) d r, \quad \alpha>-1 .
$$

Substituting for $A_{n}$ in equation (2) and interchanging the order of summation and integration we have

$$
\int_{a}^{b} e^{-r} \phi(r) S(r, x) d r=x^{\alpha} f(x), \quad a<x<b,
$$

where $S(r, x)$ is defined by equation (10).

Using the notation of equation (11) this can be written as

$$
\int_{a}^{x} e^{-r} \dot{\phi}(r) S_{r}(r, x) d r+\int_{x}^{b} e^{-r} \phi(r) S_{x}(r, x) d r=\{\Gamma(1-\beta)\}^{2} x^{\alpha} f(x),
$$

where $a<x<b, \alpha+\beta>0, \beta<1$.

Inverting the order of integration in equation (19) we find that it becomes

$$
\begin{aligned}
\int_{a}^{x} \frac{n(y)}{(x-y)^{\beta}} \Phi(y) d y & =\{\Gamma(1-\beta)\}^{2} x^{\alpha} f(x) \\
& -\int_{0}^{a} \frac{n(y)}{(x-y)^{\beta}} d y \int_{a}^{b} \frac{e^{-r} \phi(r)}{(r-y)^{\beta}} d r,
\end{aligned}
$$

where

$$
\Phi(y)=\int_{y}^{b} \frac{e^{-r} \dot{\phi}(r)}{(r-y)^{\beta}} d r .
$$

When $0<\beta<1$, equation (21) is an Abel integral equation and using the results (13) and (15), we find

$$
e^{-r} \dot{\phi}(r)=-\frac{\sin (\beta \pi)}{\pi} \frac{d}{d r} \int_{r}^{b} \frac{\Phi(y)}{(y-r)^{1-\beta}} d y .
$$

Also, from the results (12) and (14), we see that equation (20) can be written as

$$
n(y) \Phi(y)=F(y)-\frac{\sin (\beta \pi)}{\pi} \int_{0}^{a} n(\xi) l(y, \xi) d \xi \int_{a}^{b} \frac{e^{-r} \phi(r)}{(r-\xi)^{\beta}} d r,
$$

for $a+\beta>0,0<\beta<1, a<y<b$, where

$$
F(y)=\frac{\Gamma(1-\beta)}{\Gamma(\beta)} \frac{d}{d y} \int_{a}^{y} \frac{x^{\alpha} f(x)}{(y-x)^{1-\beta}} d x,
$$

is a known function, and

$$
l(y, \xi)=\frac{d}{d y} \int_{a}^{y} \frac{d x}{(y-x)^{1-\beta}(x-\xi)^{\beta}} .
$$


It is shown in [2] that

$$
l(y, \xi)=\frac{(a-\xi)^{1-\beta}}{(y-\xi)(y-a)^{1-\beta}}, \quad 0<\beta<1,
$$

and hence equation (23) becomes

$$
n(y) \Phi(y)=F(y)-\frac{\sin (\beta \pi)}{\pi(y-a)^{1-\beta}} \int_{0}^{a} \frac{(a-\xi)^{1-\beta}}{y-\xi} n(\xi) d \xi \int_{a}^{b} \frac{e^{-r} \phi(r)}{(r-\xi)^{\beta}} d r
$$

Using equation (22) we can write

$$
\begin{aligned}
\int_{a}^{b} \frac{e^{-r} \phi(r)}{(r-\xi)^{\beta}} d r & =-\frac{\sin (\beta \pi)}{\pi} \int_{a}^{b} \frac{d r}{(r-\xi)^{\beta}} \frac{d}{d r} \int_{r}^{b} \frac{\Phi(y)}{(y-r)^{1-\beta}} d y \\
& =\frac{\sin (\beta \pi)}{\pi}\left\{\frac{1}{(a-\xi)^{\beta}} \int_{a}^{b} \frac{\Phi(y)}{(y-a)^{1-\beta}} d y\right. \\
& \left.-\beta \int_{a}^{b} \frac{d r}{(r-\xi)^{1+\beta}} \int_{r}^{b} \frac{\Phi(y)}{(y-r)^{1-\beta}} d y\right\},
\end{aligned}
$$

after an integration by parts.

Inverting the order of integration in the last term of equation (28) and using the result given in [2]

$$
\beta \int_{a}^{y} \frac{d r}{(r-\xi)^{1+\beta}(y-r)^{1-\beta}}=\frac{(y-a)^{\beta}}{(y-\xi)(a-\xi)^{\beta}}, \quad 0<\beta<1,
$$

we get

$$
\int_{a}^{b} \frac{e^{-r} \phi(r)}{(r-\xi)^{\beta}} d r=\frac{\sin (\beta \pi)}{\pi(a-\xi)^{\beta-1}} \int_{a}^{b} \frac{\Phi(y)}{(y-\xi)(y-a)^{1-\beta}} d y .
$$

Substituting for this expression in equation (27) we find that $\Phi(y)$ is given by the equation

$$
n(y) \Phi(y)=F(y)-\int_{a}^{b} \Phi(x) M(x, y) d x, \quad 0<\beta<1, \quad a<y<b,
$$

where $M(x, y)$ is the symmetric kernel

$$
M(x, y)=\frac{\sin ^{2}(\beta \pi)}{\pi^{2}[(x-a)(y-a)]^{1-\beta}} \int_{0}^{a} \frac{n(\xi)(a-\xi)^{2(1-\beta)}}{(x-\xi)(y-\xi)} d \xi .
$$

Equation (31) is a Fredholm integral equation which determines $\Phi(y), \phi(r)$ is then obtained from equation (22) and the coefficients $A_{n}$ which satisfy the equations (1), (2) and (3), when $\alpha+\beta>0,0<\beta<1$, can be found from equation (17).

If we put $a=0$ the triple series equations (1), (2) and (3) reduce to the dual series equations considered in [1] and it is easily shown that the above solution reduces to the solution obtained in that paper. 
4. Equations of the second kind. To solve the triple series equations (4), (5) and (6) we put

$$
\begin{aligned}
\sum_{n=0}^{\infty} B_{n} \Gamma(\alpha+1+n) L_{n}(\alpha ; x) & =\psi_{1}(x), & & 0 \leqq x<a, \\
& =\psi_{2}(x), & & b<x<\infty,
\end{aligned}
$$

and use the orthogonality relation (9) to find that equations (5) and (33) give

(34) $\quad B_{n}=\frac{\Gamma(n+1)}{\{\Gamma(\alpha+1+n)\}^{2}}\left\{\int_{0}^{a} \psi_{1}(r)+\int_{b}^{\infty} \psi_{2}(r)\right\} r^{\alpha} e^{-r} L_{n}(\alpha ; r) d r$,

$$
\alpha>-1 \text {. }
$$

Substituting for $B_{n}$ in equations (4) and (6) and interchanging the order of summation and integration we find

$$
\begin{aligned}
\left\{\int_{0}^{a} \psi_{1}(r)+\int_{b}^{\infty} \psi_{2}(r)\right\} e^{-r} S(r, x) d r & =x^{\alpha} g(x), & & 0 \leqq x<a \\
& =x^{\alpha} h(x), & & b<x<\infty,
\end{aligned}
$$

where $S(r, x)$ is given by equation (10).

In the notation of equation (11) these equations may be written as

$$
\begin{aligned}
\int_{0}^{x} e^{-r} \psi_{1}(r) S_{r}(r, x) d r & +\int_{x}^{a} e^{-r} \psi_{1}(r) S_{x}(r, x) d r \\
& +\int_{b}^{\infty} e^{-r} \psi_{2}(r) S_{x}(r, x) d r=\{\Gamma(1-\beta)\}^{2} x^{\alpha} g(x),
\end{aligned}
$$

where $0 \leqq x<a$,

$$
\begin{aligned}
\int_{0}^{a} e^{-r} \psi_{1}(r) S_{r}(r, x) d r & +\int_{b}^{x} e^{-r} \psi_{2}(r) S_{r}(r, x) d r \\
& +\int_{x}^{\infty} e^{-r} \psi_{2}(r) S_{x}(r, x) d r=\{\Gamma(1-\beta)\}^{2} x^{\alpha} h(x),
\end{aligned}
$$

where $b<x<\infty, \alpha+\beta>0, \beta<1$.

Inverting the order of integration in the above equations we have

$$
\int_{0}^{x} \frac{n(y)}{(x-y)^{\beta}}\left\{\Psi_{1}(y)+\int_{0}^{\infty} \frac{e^{-r} \psi_{2}(r)}{(r-y)^{\beta}} d r\right\} d y=\{\Gamma(1-\beta)\}^{2} x^{\alpha} g(x)
$$

for $0 \leqq x<a$, and

$$
\begin{aligned}
\int_{b}^{x} \frac{n(y)}{(x-y)^{\beta}} \Psi_{2}(y) d y & =\{\Gamma(1-\beta)\}^{2} x^{\alpha} h(x)-\int_{0}^{a} \frac{n(y)}{(x-y)^{\beta}} \Psi_{1}(y) d y \\
& -\int_{0}^{b} \frac{n(y)}{(x-y)^{\beta}} d y \int_{b}^{\infty} \frac{e^{-r}}{(r-y)^{\beta}} \psi_{2}(r) d r
\end{aligned}
$$

where $b<x<\infty$, 


$$
\begin{aligned}
& \text { (i ) } \Psi_{1}(y)=\int_{y}^{a} \frac{e^{-r}}{(r-y)^{\beta}} \psi_{1}(r) d r, \\
& \text { (ii) } \Psi_{2}(y)=\int_{y}^{\infty} \frac{e^{-r}}{(r-y)^{\beta}} \psi_{2}(r) d r .
\end{aligned}
$$

When $0<\beta<1$ we may use the results (12) to (15) and deal with equations (38), (39) and (40) in a similar manner to that used to obtain equations (22) and (27) and find that

$$
n(y) \Psi_{1}(y)=G(y)-n(y) \int_{b}^{\infty} \frac{e^{-r} \psi_{2}(r)}{(r-y)^{\beta}} d r
$$

$$
\begin{aligned}
n(y) \Psi_{2}(y)=H(y) & -\frac{\sin (\beta \pi)}{\pi(y-b)^{1-\beta}} \int_{0}^{a} \frac{(b-\xi)^{1-\beta}}{y-\xi} n(\xi) \Psi_{1}(\xi) d \xi \\
& -\frac{\sin (\beta \pi)}{\pi(y-b)^{1-\beta}} \int_{0}^{b} \frac{(b-\xi)^{1-\beta}}{y-\xi} n(\xi) d \xi \int_{b}^{\infty} \frac{e^{-r} \psi_{2}(r)}{(r-\xi)^{\beta}} d r,
\end{aligned}
$$

$$
e^{-r} \psi_{1}(r)=-\frac{\sin (\beta \pi)}{\pi} \frac{d}{d r} \int_{r}^{a} \frac{\Psi_{1}(y)}{(y-r)^{1-\beta}} d y, \quad 0<r<a,
$$

and

$$
e^{-r} \psi_{2}(r)=-\frac{\sin (\beta \pi)}{\pi} \frac{d}{d r} \int_{r}^{\infty} \frac{\Psi_{2}(y)}{(y-r)^{1-3}} d y, \quad b<r<\infty,
$$

where $G(y)$ and $H(y)$ are the known functions

$$
G(y)=\frac{\Gamma(1-\beta)}{\Gamma(\beta)} \frac{d}{d y} \int_{0}^{y} \frac{x^{\alpha} g(x)}{(y-x)^{1-\beta}} d x, \quad 0<y<a,
$$

and

$$
H(y)=\frac{\Gamma(1-\beta)}{\Gamma(\beta)} \frac{d}{d y} \int_{b}^{y} \frac{x^{\alpha} h(x)}{(y-x)^{1-\beta}} d x, \quad b<y<\infty .
$$

From equation (41), using the result (43), it can be shown that $\psi_{1}(r)$ and $\psi_{2}(r)$ are related by the equation

$$
\begin{aligned}
e^{-r} \psi_{1}(r) & =-\frac{\sin (\beta \pi)}{\pi} \frac{d}{d r} \int_{r}^{a} \frac{G(y)}{n(y)(y-r)^{1-\beta}} \mathrm{d} y \\
& +\frac{\sin (\beta \pi)}{\pi(a-r)^{1-\beta}} \int_{b}^{\infty} \frac{e^{-\hat{\xi}}(\xi-a)^{1-\beta}}{r-\xi} \psi_{2}(\xi) d \xi,
\end{aligned}
$$

where $0<r<a$.

By a similar method to that used to obtain equation (30) we can show that

$$
\int_{b}^{\infty} \frac{e^{-r} \psi_{2}(r)}{(r-y)^{3}} d r=\frac{\sin (\beta \pi)}{\pi(b-y)^{\beta-1}} \int_{b}^{\infty} \frac{(\xi-b)^{\beta-1}}{\xi-y} \Psi_{2}(\xi) d \xi .
$$


Using this result and equation (41) it can be shown, after some manipulation, that equation (42) can be written in the form

$$
\begin{aligned}
n(y) \Psi_{2}(y) & +\int_{b}^{\infty} \Psi_{2}(x) N(x, y) d x \\
& =H(y)-\frac{\sin (\beta \pi)}{\pi(y-b)^{1-\beta}} \int_{0}^{a} \frac{(b-\xi)^{1-\beta}}{y-\xi} G(\xi) d \xi,
\end{aligned}
$$

where $N(x, y)$ is the symmetric kernel

$$
N(x, y)=\frac{\sin ^{2}(\beta \pi)}{\pi^{2}[(x-b)(y-b)]^{1-\beta}} \int_{a}^{b} \frac{n(\xi)(b-\xi)^{2(1-\beta)}}{(x-\xi)(y-\xi)} d \xi, \quad b<y<\infty .
$$

Equation (49) is a Fredholm integral equation of the second kind which determines $\Psi_{2}(y), \psi_{2}(r)$ can then be found from equation (44) and $\psi_{1}(r)$ from equation (47). Finally, the coefficients $B_{n}$ which satisfy the triple series equations of the second kind when $\alpha+\beta>0$, $0<\beta<1$, are given by equation (34).

If we let $b \rightarrow \infty$ in equations (4), (5) and (6) they reduce to the dual series equations considered in [1] and the above solution can be shown to agree with the solution obtained in that paper.

\section{REFERENCES}

1. J. S. Lowndes, Some dual series equations involving Laguerre polynomials, Pacific J. Math. 25 (1968), 123-127.

2. - Dual series and triple integral equations Proc. Edin. Math. Soc. (to be published)

3. — Some triple series equations involving Jacobi polynomials, Proc. Edin. Math. Soc. 16 (1968), 101-108.

4. I. N. Sneddon, Mixed boundary value problems in potential theory, North-Holland, 1966.

5. K. N. Srivastava, On triple series equations involving series of Jacobi polynomials, Proc. Edin. Math. Soc. 15 (1967), 221-233.

Received October 2, 1967,

UNIVERSITY OF STRATHCLYDE

GLASGOW, Scotland 




\section{Pacific Journal of Mathematics \\ Vol. 29, No. 1 \\ May, 1969}

Jorge Alvarez de Araya, A Radon-Nikodým theorem for vector and operator

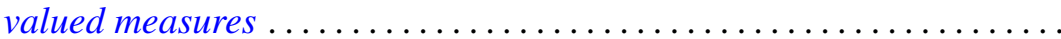

Deane Eugene Arganbright, The power-commutator structure of finite

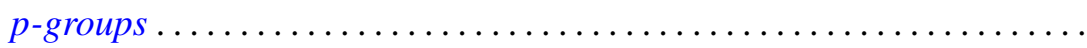

Richard Eugene Barlow, Albert W. Marshall and Frank Proschan, Some

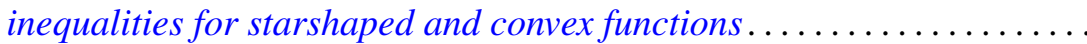

David Clarence Barnes, Some isoperimetric inequalities for the eigenvalues

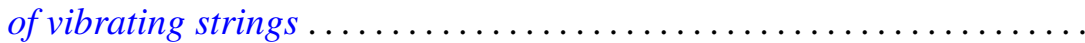

David Hilding Carlson, Critical points on rim-compact spaces ...........

Allan Matlock Weber Carstens, The lattice of pretopologies on an arbitrary

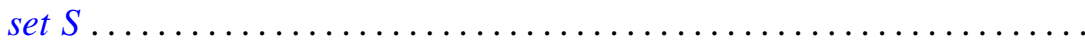

S. K. Chatterjea, A bilateral generating function for the ultraspherical

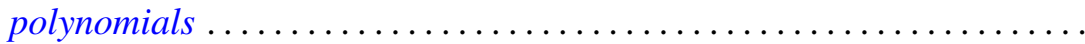

Ronald J. Ensey, Primary Abelian groups modulo finite groups ......... 77

Harley M. Flanders, Relations on minimal hypersurfaces ............ 83

Allen Roy Freedman, On asymptotic density in n-dimensions........... 95

Kent Ralph Fuller, On indecomposable injectives over artinian rings...... 115

George Isaac Glauberman, Normalizers of p-subgroups in finite groups . . . 137

William James Heinzer, On Krull overrings of an affine ring ........... 145

John McCormick Irwin and Takashi Ito, A quasi-decomposable abelian group without proper isomorphic quotient groups and proper isomorphic subgroups.

Allan Morton Krall, Boundary value problems with interior point boundary conditions

John S. Lowndes, Triple series equations involving Laguerre

polynomials

Philip Olin, Indefinability in the arithmetic isolic integers

Ki-Choul Oum, Bounds for the number of deficient values of entire functions whose zeros have angular densities..

R. D. Schafer, Standard algebras ....................

Wolfgang M. Schmidt, Irregularities of distribution. III.

Richard Alfred Tapia, An application of a Newton-like method to the Euler-Lagrange equation 